\title{
The uniqueness of solution for a fractional order nonlinear eigenvalue problem with $p$-Laplacian operator
}

\section{Ge-Feng Yang* and Minling Zhong}

"Correspondence:

yanggefeng_001@163.com Cisco School of Informatics,

Guangdong University of Foreign

Studies, Guangzhou, 510006, China

\begin{abstract}
In this article, we investigate the uniqueness of solutions for the fractional order differential equation with $p$-Laplacian operator $-\mathscr{D}_{t}^{\alpha}\left(\varphi_{p}\left(\mathscr{D}_{t}^{\beta} x\right)\right)(t)=\lambda f(t, x(t)), t \in(0,1)$, $x(0)=0, \mathscr{D}_{t}^{\beta} x(0)=0, \mathscr{D}_{t}^{\gamma} x(1)=\sum_{j=1}^{m-2} a_{j} \mathscr{D}_{t}^{\gamma} x\left(\xi_{j}\right)$, where $\mathscr{D}_{t}^{\alpha}, \mathscr{D}_{t}^{\beta}, \mathscr{D}_{t}^{\gamma}$ are the standard Riemann-Liouville derivatives with $1<\beta \leq 2,0<\alpha \leq 1,0<\gamma \leq 1,0 \leq \beta-\gamma-1$, $0<\xi_{1}<\xi_{2}<\cdots<\xi_{p-2}<1, a_{j} \in[0,+\infty)$ with $c=\sum_{j=1}^{m-2} a_{j} \xi_{j}^{\beta-\gamma-1}<1$, and the $p$-Laplacian operator is defined as $\varphi_{p}(s)=|s|^{p-2} s, p>1$. Based on a basic property of the $p$-Laplacian operator and the Banach contraction mapping principle, the uniqueness of solutions for the fractional order differential equation is established for the cases $p>2$ and $1<p \leq 2$.
\end{abstract}

MSC: $26 \mathrm{~A} 33 ; 34 \mathrm{~B} 10$

Keywords: eigenvalue problem; uniqueness; $p$-Laplacian operator; fractional differential equation

\section{Introduction}

Differential equations of fractional order have been shown to be valuable tools in the modeling of many phenomena arising from science and engineering, such as the charge transport in amorphous semiconductors [1], flows through porous media, electrochemistry and material science [2-5]. In the recent years, there has been a significant development in fractional order differential equations involving various boundary conditions. For example, by using the contraction mapping principle, Rehman and Khan [6] established the existence and uniqueness of positive solutions for the following fractional order multi-point boundary value problem:

$$
\left\{\begin{array}{l}
\mathscr{D}_{t}^{\alpha} y(t)=f\left(t, y(t), \mathscr{D}_{t}^{\beta} y(t)\right), \quad t \in(0,1), \\
y(0)=0, \quad \mathscr{D}_{t}^{\beta} y(1)-\sum_{i=1}^{m-2} \zeta_{i} \mathscr{D}_{t}^{\beta} y\left(\xi_{i}\right)=y_{0},
\end{array}\right.
$$

where $1<\alpha \leq 2,0<\beta<1, \zeta_{i} \in[0,+\infty), 0<\xi_{i}<1$, with $\sum_{i=1}^{m-2} \zeta_{i} \xi_{i}<1$. In [7], Zhang et al. discussed the existence and uniqueness of positive solutions for the following fractional differential equation with derivatives:

$$
\left\{\begin{array}{l}
-\mathscr{D}_{t}^{\alpha} x(t)=f\left(t, x(t),-\mathscr{D}_{t}^{\beta} x(t)\right), \quad t \in(0,1), \\
\mathscr{D}_{t}^{\beta} x(0)=0, \quad \mathscr{D}_{t}^{\gamma} x(1)=\sum_{j=1}^{p-2} a_{j} \mathscr{D}_{t}^{\gamma} x\left(\xi_{j}\right),
\end{array}\right.
$$

( 2014 Yang and Zhong; licensee Springer. This is an Open Access article distributed under the terms of the Creative Commons Attribution License (http://creativecommons.org/licenses/by/2.0), which permits unrestricted use, distribution, and reproduction in any medium, provided the original work is properly cited. 
where $1<\alpha \leq 2, \alpha-\beta>1,0<\beta \leq \gamma<1,0<\xi_{1}<\xi_{2}<\cdots<\xi_{p-2}<1, a_{j} \in[0,+\infty)$ with $c=$ $\sum_{j=1}^{p-2} a_{j} \xi_{j}^{\alpha-\gamma-1}<1, \mathscr{D}_{t}^{\alpha}$ is the standard Riemann-Liouville derivative. $f:(0,1) \times[0,+\infty) \times$ $(-\infty,+\infty) \rightarrow[0,+\infty)$ is continuous, and $f(t, u, v)$ may be singular at $t=0,1$. By means of monotone iterative technique, the existence and uniqueness of the positive solution for a fractional differential equation with derivatives are established, and the iterative sequence of the solution, an error estimation and the convergence rate of the positive solution are also given.

Recently, some excellent work on nonlocal integral boundary condition for fractional differential equation and system was done by Zhang et al. [8] and Ahmad and Nieto [9]. In [8], by establishing some comparison results and combining with a monotone iterative method, the existence of an extremal solution for a nonlinear system involving the righthanded Riemann-Liouville fractional derivative with nonlocal coupled integral boundary conditions was obtained. Ahmad and Nieto [9] employed standard fixed point theorems to study the uniqueness and existence of solution for a class of Riemann-Liouville fractional differential equations with fractional boundary conditions. Some new existence and uniqueness results are obtained. Here we also refer the reader to some recent work on fractional differential equation (see [10-17]).

Since the turbulent flow in a porous medium is a fundamental mechanics problem, Leibenson [18] introduced the following $p$-Laplacian equation to describe the flow of porous medium:

$$
\left(\varphi_{p}\left(x^{\prime}(t)\right)\right)^{\prime}=f\left(t, x(t), x^{\prime}(t)\right)
$$

where $\varphi_{p}(s)=|s|^{p-2} s, p>1$. Inspired by Leibenson's work, Wang et al. [14] investigated the nonlinear nonlocal boundary value problem

$$
\left\{\begin{array}{l}
\mathscr{D}_{t}^{\alpha}\left(\varphi_{p}\left(\mathscr{D}_{t}^{\beta} x\right)\right)(t)+f(t, x(t))=0, \\
x(0)=0, \quad \mathscr{D}_{t}^{\beta} x(0)=0, \quad x(1)=a x(\xi),
\end{array}\right.
$$

where $0<\beta \leq 2,0<\alpha \leq 1,0 \leq a \leq 1,0<\xi<1$. By using Krasnosel'skii's fixed point theorem and the Leggett-Williams theorem, some sufficient conditions for the existence of positive solutions to the above BVP are obtained. Then, by using upper and lower solutions method, Wu and Zhou [12] studied the existence of positive solutions for the fractional order eigenvalue problem with the $p$-Laplacian operator

$$
\left\{\begin{array}{l}
-\mathscr{D}_{t}^{\alpha}\left(\varphi_{p}\left(\mathscr{D}_{t}^{\beta} x\right)\right)(t)=\lambda f(t, x(t)), \quad t \in(0,1), \\
x(0)=0, \quad \mathscr{D}_{t}^{\beta} x(0)=0, \quad \mathscr{D}_{t}^{\gamma} x(1)=\sum_{j=1}^{m-2} a_{j} \mathscr{D}_{t}^{\gamma} x\left(\xi_{j}\right),
\end{array}\right.
$$

where $\mathscr{D}_{t}^{\alpha}, \mathscr{D}_{t}^{\beta}, \mathscr{D}_{t}^{\gamma}$ are the standard Riemann-Liouville derivatives with $1<\beta \leq 2,0<\alpha \leq$ $1,0<\gamma \leq 1,0 \leq \beta-\gamma-1,0<\xi_{1}<\xi_{2}<\cdots<\xi_{p-2}<1, a_{j} \in[0,+\infty)$ with $c=\sum_{j=1}^{m-2} a_{j} \xi_{j}^{\beta-\gamma-1}<$ 1 , the $p$-Laplacian operator is defined as $\varphi_{p}(s)=|s|^{p-2} s, p>1$. By constructing upper and lower solutions, the existence of positive solutions for the problem is established.

However, because of the stronger nonlinearity of the $p$-Laplacian operator, the uniqueness of solution for the above problem is still unknown. It is well known that the Banach contraction mapping principle is difficult to apply to the $p$-Laplacian operator to obtain the uniqueness of solution since it is nonlinear. In this paper, by studying the property 
of the $p$-Laplacian operator, we overcome this difficulty and establish the uniqueness of solution for the eigenvalue problem of the fractional differential equation (1.1).

The rest of this article is organized as follows. In Section 2, we present some definitions and preliminary results that are to be used to prove our main results. In Section 3, we present our main results followed by the proofs. Finally, we give an example to demonstrate the application of our main results.

\section{Preliminaries and lemmas}

In this paper, we restrict our attention to the use of the Riemann-Liouville fractional derivatives. For details of some basic definitions of the fractional calculus, we refer the reader to [19-21] or other texts on basic fractional calculus.

Based on a basic fact of the $p$-Laplacian operator, we can obtain the following lemma.

Lemma 2.1 (1) If $q \geq 2,|x|,|y| \leq M$, then

$$
\left|\varphi_{q}(x)-\varphi_{q}(y)\right| \leq(q-1) M^{q-2}|x-y| .
$$

(2) If $1<q<2, x y>0$, and $|x|,|y| \geq m>0$, then

$$
\left|\varphi_{q}(x)-\varphi_{q}(y)\right| \leq(q-1) m^{q-2}|x-y| .
$$

Lemma 2.2 (see [17]) Suppose that $h \in L^{1}[0,1]$. Then the following boundary value problem:

$$
\left\{\begin{array}{l}
\mathscr{D}_{t}^{\beta} x(t)+h(t)=0, \quad t \in(0,1), \\
x(0)=0, \quad \mathscr{D}_{t}^{\gamma} x(1)=\sum_{j=1}^{m-2} a_{j} \mathscr{D}_{t}^{\gamma} x\left(\xi_{j}\right)
\end{array}\right.
$$

is equivalent to the following integral equation:

$$
x(t)=\int_{0}^{1} G(t, s) h(s) d s,
$$

where

$$
G(t, s)=g_{1}(t, s)+\frac{t^{\beta-1}}{1-\sum_{j=1}^{m-2} a_{j} \xi_{j}^{\beta-\gamma-1}} \sum_{j=1}^{m-2} a_{j} g_{2}\left(\xi_{j}, s\right),
$$

is the Green function of the boundary value problem (2.3) and

$$
\begin{aligned}
& g_{1}(t, s)= \begin{cases}\frac{t^{\beta-1}(1-s)^{\beta-\gamma-1}-(t-s)^{\beta-1}}{\Gamma(\beta)}, & 0 \leq s \leq t \leq 1, \\
\frac{t^{\beta-1}(1-s)^{\beta-\gamma-1}}{\Gamma(\beta)}, & 0 \leq t \leq s \leq 1,\end{cases} \\
& g_{2}(t, s)= \begin{cases}\frac{(t(1-s))^{\beta-\gamma-1}-(t-s)^{\beta-\gamma-1}}{\Gamma(\beta)}, & 0 \leq s \leq t \leq 1, \\
\frac{(t(1-s))^{\beta-\gamma-1}}{\Gamma(\beta)}, & 0 \leq t \leq s \leq 1 .\end{cases}
\end{aligned}
$$

Lemma 2.3 The Green function $G(t, s)$ in Lemma 2.2 has the following properties:

(i) $G(t, s)$ is continuous on $[0,1] \times[0,1]$ and $G(t, s)>0$ for any $s, t \in(0,1)$; 
(ii)

$$
G(t, s) \leq \frac{r}{\Gamma(\beta)}(1-s)^{\beta-\gamma-1}, \quad \text { for } t, s \in[0,1]
$$

where

$$
r=1+\frac{\sum_{j=1}^{m-2} a_{j}}{1-\sum_{j=1}^{m-2} a_{j} \xi_{j}^{\beta-\gamma-1}}
$$

Proof (i) is obvious. We prove that (ii) is valid.

In fact, by (2.6), we have

$$
g_{i}(t, s) \leq \frac{(1-s)^{\beta-\gamma-1}}{\Gamma(\beta)}, \quad s, t \in[0,1], i=1,2 .
$$

Equation (2.7) is a straightforward consequence of (2.8). The proof is thus completed.

Let $q>1$ satisfy the relation $\frac{1}{q}+\frac{1}{p}=1$. We consider the associated linear boundary value problem

$$
\left\{\begin{array}{l}
\mathscr{D}_{t}^{\alpha}\left(\varphi_{p}\left(\mathscr{D}_{t}^{\beta} x\right)\right)(t)+h(t)=0, \quad t \in(0,1), \\
x(0)=0, \quad \mathscr{D}_{t}^{\beta} x(0)=0, \quad \mathscr{D}_{t}^{\gamma} x(1)=\sum_{j=1}^{m-2} a_{j} \mathscr{D}_{t}^{\gamma} x\left(\xi_{j}\right),
\end{array}\right.
$$

for $h \in L^{1}[0,1]$ and $h \geq 0$.

Lemma 2.4 (see [14]) The associated linear boundary value problem (2.9) has unique positive solution

$$
x(t)=\int_{0}^{1} G(t, s) \varphi_{q}\left(\frac{1}{\Gamma(\alpha)} \int_{0}^{s}(s-\tau)^{\alpha-1} h(\tau) d \tau\right) d s .
$$

Let the Banach space $E=C[0,1]$ be endowed with the norm $\|x\|:=\max _{t \in[0,1]}|x(t)|$. By Lemma $2.4, x \in E$ is a solution of the boundary value problem (1.1) if and only if $x \in E$ is a solution of the integral equation

$$
x(t)=\lambda \int_{0}^{1} G(t, s) \varphi_{q}\left(\frac{1}{\Gamma(\alpha)} \int_{0}^{s}(s-\tau)^{\alpha-1} f(\tau, x(\tau)) d \tau\right) d s .
$$

We define an operator $T: E \rightarrow E$ by

$$
T x(t)=\lambda \int_{0}^{1} G(t, s) \varphi_{q}\left(\frac{1}{\Gamma(\alpha)} \int_{0}^{s}(s-\tau)^{\alpha-1} f(\tau, x(\tau)) d \tau\right) d s .
$$

It is easy to see that $x$ is the solution of the boundary value problem (1.1) if and only if $x$ is the fixed point of $T$. As $f \in C([0,1] \times \mathbb{R}, \mathbb{R})$, we know that $T: E \rightarrow E$ is a continuous and compact operator.

\section{Main results}

In this section, we use the Banach contraction mapping principle to prove the existence and uniqueness of the solution of problem (1.1). Firstly, we give the result on the case $p>2$. As $\frac{1}{p}+\frac{1}{q}=1$, if $p>2$, we have $1<q<2$, and we have the following theorem. 
Theorem 3.1 Suppose $p>2$, and the following conditions hold:

$\left(\mathrm{A}_{1}\right)$ There exists a nonnegative continuous function $a(t)$, which does not vanish for some $t_{0} \in(0,1)$, and a constant $\mu>0$ such that for any $c \in(0,1)$

$$
a(c t) \geq c^{\mu} a(t), \quad t \in[0,1]
$$

$\left(\mathrm{A}_{2}\right)$ For any $(t, x) \in(0,1] \times \mathbb{R}$,

$$
f(t, x) \geq a(t)
$$

$\left(\mathrm{A}_{3}\right)$ There exists some constant $\kappa \in(0, \alpha)$ such that $b(t) \in L^{\frac{1}{\kappa}}([0,1],[0,+\infty))$ and

$$
|f(t, x)-f(t, y)| \leq b(t)|x-y|, \quad \text { a.e. }(t, x) \in[0,1] \times \mathbb{R} .
$$

Then there exists a constant $\Lambda>0$ such that for any $\lambda \in(0, \Lambda)$, the $B V P(1.1)$ has a unique solution.

Proof Let

$$
\begin{aligned}
\Lambda= & \left\{\frac{(q-1) r\|b\|_{L^{\frac{1}{\kappa}}} B(\beta-\gamma,(\alpha+\mu)(q-2)+1)}{\Gamma(\beta) \Gamma^{q-1}(\alpha)}\right. \\
& \left.\times\left(\int_{0}^{1}(1-\tau)^{\alpha-1} a(\tau) d \tau\right)^{q-2}\left(\frac{1-\kappa}{\alpha-\kappa}\right)^{1-\kappa}\right\}^{-1} .
\end{aligned}
$$

For any $\lambda \in(0, \Lambda)$, we shall prove that $F$ is a contraction mapping. By (3.1) and (3.2), we have

$$
\begin{aligned}
\int_{0}^{s}(s-\tau)^{\alpha-1} f(\tau, x(\tau)) d \tau & \geq \int_{0}^{s}(s-\tau)^{\alpha-1} a(\tau) d \tau=\int_{0}^{1}(1-\tau)^{\alpha-1} s^{\alpha} a(s \tau) d \tau \\
& \geq s^{\alpha+\mu} \int_{0}^{1}(1-\tau)^{\alpha-1} a(\tau) d \tau .
\end{aligned}
$$

By (2.2), (3.3), and (3.5), for any $x, y \in E$ and for $t>0$, we have

$$
\begin{aligned}
& \left|\varphi_{q}\left(\frac{1}{\Gamma(\alpha)} \int_{0}^{s}(s-\tau)^{\alpha-1} f(\tau, x(\tau)) d \tau\right)-\varphi_{q}\left(\frac{1}{\Gamma(\alpha)} \int_{0}^{s}(s-\tau)^{\alpha-1} f(\tau, x(\tau)) d \tau\right)\right| \\
& \quad \leq \frac{q-1}{\Gamma^{q-1}(\alpha)}\left(s^{\alpha+\mu} \int_{0}^{1}(1-\tau)^{\alpha-1} a(\tau) d \tau\right)^{q-2} \int_{0}^{s}(s-\tau)^{\alpha-1}|f(\tau, x(\tau))-f(\tau, y(\tau))| d \tau \\
& \quad \leq \frac{q-1}{\Gamma^{q-1}(\alpha)}\left(s^{\alpha+\mu} \int_{0}^{1}(1-\tau)^{\alpha-1} a(\tau) d \tau\right)^{q-2} \int_{0}^{s}(s-\tau)^{\alpha-1} b(\tau)|x-y| d \tau \\
& \leq \frac{q-1}{\Gamma^{q-1}(\alpha)}\left(s^{\alpha+\mu} \int_{0}^{1}(1-\tau)^{\alpha-1} a(\tau) d \tau\right)^{q-2}\left(\frac{1-\kappa}{\alpha-\kappa}\right)^{1-\kappa}\|b\|_{L^{\frac{1}{\kappa}}}\|x-y\| \\
& \quad=\frac{(q-1) s^{(\alpha+\mu)(q-2)}\|b\|_{L^{\frac{1}{\kappa}}}}{\Gamma^{q-1}(\alpha)}\left(\int_{0}^{1}(1-\tau)^{\alpha-1} a(\tau) d \tau\right)^{q-2}\left(\frac{1-\kappa}{\alpha-\kappa}\right)^{1-\kappa}\|x-y\| .
\end{aligned}
$$


So it follows from (2.7) and (3.5) that

$$
\begin{aligned}
& |F x(t)-F y(t)| \\
& =\mid \lambda \int_{0}^{1} G(t, s)\left[\varphi_{q}\left(\frac{1}{\Gamma(\alpha)} \int_{0}^{s}(s-\tau)^{\alpha-1} f(\tau, x(\tau)) d \tau\right) d s\right. \\
& \left.-\varphi_{q}\left(\frac{1}{\Gamma(\alpha)} \int_{0}^{s}(s-\tau)^{\alpha-1} f(\tau, y(\tau)) d \tau\right)\right] d s \mid \\
& \leq \frac{\lambda r}{\Gamma(\beta)} \int_{0}^{1}(1-s)^{\beta-\gamma-1} \mid \varphi_{q}\left(\frac{1}{\Gamma(\alpha)} \int_{0}^{s}(s-\tau)^{\alpha-1} f(\tau, x(\tau)) d \tau\right) d s \\
& -\varphi_{q}\left(\frac{1}{\Gamma(\alpha)} \int_{0}^{s}(s-\tau)^{\alpha-1} f(\tau, y(\tau)) d \tau\right) \mid d s \\
& \leq \frac{\lambda(q-1) r\|b\|_{L^{\frac{1}{\kappa}}}}{\Gamma(\beta) \Gamma^{q-1}(\alpha)}\left(\int_{0}^{1}(1-\tau)^{\alpha-1} a(\tau) d \tau\right)^{q-2} \\
& \times\left(\frac{1-\kappa}{\alpha-\kappa}\right)^{1-\kappa} \int_{0}^{1}(1-s)^{\beta-\gamma-1} s^{(\alpha+\mu)(q-2)} d s\|x-y\| \\
& \leq \frac{\lambda(q-1) r\|b\|_{L^{\frac{1}{\kappa}}} B(\beta-\gamma,(\alpha+\mu)(q-2)+1)}{\Gamma(\beta) \Gamma^{q-1}(\alpha)} \\
& \times\left(\int_{0}^{1}(1-\tau)^{\alpha-1} a(\tau) d \tau\right)^{q-2}\left(\frac{1-\kappa}{\alpha-\kappa}\right)^{1-\kappa}\|x-y\|,
\end{aligned}
$$

which implies that

$$
\|F x-F y\| \leq \lambda \Lambda^{-1}\|x-y\|
$$

and then $F: C[0,1] \rightarrow C[0,1]$ is a contraction mapping since $0<\lambda \Lambda^{-1}<1$. By means of the Banach contraction mapping principle, we get the result that $F$ has a unique fixed point in $C[0,1]$, that is, the BVP $(1.1)$ has a unique solution.

In the case $1<p \leq 2$, as $\frac{1}{p}+\frac{1}{q}=1$, we get $q \geq 2$, and we have the following theorem.

Theorem 3.2 Suppose $1<p \leq 2$, and the following condition holds:

$\left(\mathrm{A}_{4}\right)$ There exist some constant $\kappa \in(0, \alpha)$ and functions $c(t), d(t)$ such that $c(t), d(t) \in$ $L^{\frac{1}{\kappa}}([0,1],[0,+\infty))$, and

$$
\begin{aligned}
& |f(t, x)| \leq c(t) \quad \text { and } \\
& |f(t, x)-f(t, y)| \leq d(t)|x-y|, \quad \text { a.e. }(t, x) \in[0,1] \times \mathbb{R} .
\end{aligned}
$$

Then there exists a constant $\Lambda>0$ such that for any $\lambda \in(0, \Lambda)$, the $B V P(1.1)$ has a unique solution.

Proof In this case, we choose

$$
\Lambda=\left\{\frac{(q-1) r\|c\|_{L^{\frac{1}{\kappa}}}^{q-1}\|d\|_{L^{\frac{1}{\kappa}}}}{(\beta-\gamma) \Gamma(\beta) \Gamma^{q-2}(\alpha)}\left(\frac{1-\kappa}{\alpha-\kappa}\right)^{(1-\kappa)(q-1)}\right\}^{-1}
$$


and prove that $F$ is a contraction mapping for any $\lambda \in(0, \Lambda)$. It follows from (3.7) and the Hölder inequality that

$$
\left|\int_{0}^{s}(s-\tau)^{\alpha-1} f(\tau, x(\tau)) d \tau\right| \leq \int_{0}^{s}(s-\tau)^{\alpha-1} c(\tau) d \tau \leq\left(\frac{1-\kappa}{\alpha-\kappa}\right)^{1-\kappa}\|c\|_{L^{\frac{1}{\kappa}}} .
$$

From (2.1) and (3.9), for any $x, y \in E$,

$$
\begin{aligned}
\mid \varphi_{q} & \left(\frac{1}{\Gamma(\alpha)} \int_{0}^{s}(s-\tau)^{\alpha-1} f(\tau, x(\tau)) d \tau\right)-\varphi_{q}\left(\frac{1}{\Gamma(\alpha)} \int_{0}^{s}(s-\tau)^{\alpha-1} f(\tau, x(\tau)) d \tau\right) \mid \\
\leq & (q-1)\left(\frac{1-\kappa}{\alpha-\kappa}\right)^{(1-\kappa)(q-2)}\left(\frac{1}{\Gamma(\alpha)}\right)^{q-1} \\
& \times\|c\|_{L^{\frac{1}{\kappa}}}^{q-2} \int_{0}^{s}(s-\tau)^{\alpha-1}|f(\tau, x(\tau))-f(\tau, y(\tau))| d \tau \\
\leq & (q-1)\left(\frac{1-\kappa}{\alpha-\kappa}\right)^{(1-\kappa)(q-2)}\left(\frac{1}{\Gamma(\alpha)}\right)^{q-1}\|c\|_{L^{\frac{1}{\kappa}}}^{q-2} \int_{0}^{s}(s-\tau)^{\alpha-1} d(\tau)|x-y| d \tau \\
\leq & (q-1)\left(\frac{1-\kappa}{\alpha-\kappa}\right)^{(1-\kappa)(q-1)}\left(\frac{1}{\Gamma(\alpha)}\right)^{q-1}\|c\|_{L^{\frac{1}{\kappa}}}^{q-2}\|d\|_{L^{\frac{1}{\kappa}}}\|x-y\| \\
\leq & (q-1)\left(\frac{1-\kappa}{\alpha-\kappa}\right)^{(1-\kappa)(q-1)}\left(\frac{1}{\Gamma(\alpha)}\right)^{q-1}\|c\|_{L^{\frac{1}{\kappa}}}^{q-2}\|d\|_{L^{\frac{1}{\kappa}}}\|x-y\|=: L\|x-y\| .
\end{aligned}
$$

So by (2.7) and (3.10), we have

$$
\begin{aligned}
\mid F x(t) & -F y(t) \mid \\
= & \mid \lambda \int_{0}^{1} G(t, s)\left[\varphi_{q}\left(\frac{1}{\Gamma(\alpha)} \int_{0}^{s}(s-\tau)^{\alpha-1} f(\tau, x(\tau)) d \tau\right)\right. \\
& \left.-\varphi_{q}\left(\frac{1}{\Gamma(\alpha)} \int_{0}^{s}(s-\tau)^{\alpha-1} f(\tau, y(\tau)) d \tau\right)\right] d s \mid \\
\leq & \frac{\lambda r}{\Gamma(\beta)} \int_{0}^{1}(1-s)^{\beta-\gamma-1} \mid \varphi_{q}\left(\frac{1}{\Gamma(\alpha)} \int_{0}^{s}(s-\tau)^{\alpha-1} f(\tau, x(\tau)) d \tau\right) \\
& -\varphi_{q}\left(\frac{1}{\Gamma(\alpha)} \int_{0}^{s}(s-\tau)^{\alpha-1} f(\tau, y(\tau)) d \tau\right) \mid d s \\
\leq & \frac{\lambda(q-1) r\|c\|_{L^{\frac{1}{\kappa}}}^{q-1}\|d\|_{L^{\frac{1}{\kappa}}}}{\Gamma(\beta) \Gamma^{q-2}(\alpha)}\left(\frac{1-\kappa}{\alpha-\kappa}\right)^{(1-\kappa)(q-1)} \int_{0}^{1}(1-s)^{\beta-\gamma-1} d s\|x-y\| \\
= & \frac{\lambda(q-1) r\|c\|_{L^{\frac{1}{\kappa}}}^{q-1}\|d\|_{L^{\frac{1}{\kappa}}}}{(\beta-\gamma) \Gamma(\beta) \Gamma^{q-2}(\alpha)}\left(\frac{1-\kappa}{\alpha-\kappa}\right)^{(1-\kappa)(q-1)}\|x-y\|,
\end{aligned}
$$

which implies that

$$
\|F x-F y\| \leq \lambda \Lambda^{-1}\|x-y\|,
$$

and thus $F: C[0,1] \rightarrow C[0,1]$ is a contraction mapping since $0<\lambda \Lambda^{-1}<1$. By means of the Banach contraction mapping principle, we get the result that $F$ has a unique fixed point in $C[0,1]$, that is, the BVP $(1.1)$ has a unique solution. 
Example Consider the following fractional order differential equation with the $p$-Laplacian operator:

$$
\left\{\begin{array}{l}
-\mathscr{D}_{t}^{\frac{1}{3}}\left(\varphi_{3}\left(\mathscr{D}_{t}^{\frac{3}{2}} x\right)\right)(t)=\lambda\left(t^{\frac{1}{8}}+\frac{\sin ^{4} x}{t^{\frac{1}{10}}}\right), \quad t \in(0,1), \\
x(0)=0, \quad \mathscr{D}_{t}^{\frac{3}{2}} x(0)=0, \quad \mathscr{D}_{t}^{\frac{1}{6}} x(1)=\frac{1}{4} \mathscr{D}_{t}^{\frac{1}{6}} x\left(\frac{1}{3}\right)+\frac{3}{4} \mathscr{D}_{t}^{\frac{1}{6}} x\left(\frac{1}{2}\right) .
\end{array}\right.
$$

The BVP (3.11) has a unique solution for any $\lambda \in(0,0.1227)$.

Proof Let

$$
\beta=\frac{3}{2}, \quad \alpha=\frac{1}{3}, \quad \gamma=\frac{1}{6}, \quad f(t, x)=t^{\frac{1}{8}}+\frac{\sin ^{4} x}{t^{\frac{1}{10}}},
$$

and choose

$$
a(t)=t^{\frac{1}{8}}, \quad b(t)=4 t^{-\frac{1}{10}}, \quad \mu=\frac{1}{6},
$$

then we have

$$
|f(t, x)-f(t, y)| \leq 4 t^{-\frac{1}{10}}|x-y|=b(t)|x-y|
$$

and $\Lambda=0.1227$. Thus $\left(\mathrm{A}_{1}\right)-\left(\mathrm{A}_{3}\right)$ all are satisfied, by Theorem 3.1, the BVP (3.11) has a unique solution for any $\lambda \in(0,0.1227)$.

\section{Competing interests}

The authors declare that there is no conflict of interests regarding the publication of this paper.

\section{Authors' contributions}

All authors contributed equally to the writing of this paper. All authors read and approved the final manuscript.

Received: 5 May 2014 Accepted: 26 June 2014 Published: 22 Jul 2014

\section{References}

1. Scher, H, Montroll, E: Anomalous transit-time dispersion in amorphous solids. Phys. Rev. B 12, 2455-2477 (1975)

2. Sabatier, J, Agrawal, O, Machado, J: Advances in Fractional Calculus: Theoretical Developments and Applications in Physics and Engineering. Springer, Dordrecht (2007)

3. Gaul, L, Klein, P, Kemple, S: Damping description involving fractional operators. Mech. Syst. Signal Process. 5, 81-88 (1991)

4. Glockle, W, Nonnenmacher, T: A fractional calculus approach to self-similar protein dynamics. Biophys. J. 68, 46-53 (1995)

5. Metzler, R, Schick, W, Kilian, H, Nonnenmacher, T: Relaxation in filled polymers: a fractional calculus approach. J. Chem. Phys. 103, 7180-7186 (1995)

6. Rehman, M, Khan, R: Existence and uniqueness of solutions for multi-point boundary value problems for fractional differential equations. Appl. Math. Lett. 23, 1038-1044 (2010)

7. Zhang, $X, L i u, L, W u, Y, L u, Y$ : The iterative solutions of nonlinear fractional differential equations. Appl. Math. Comput. 219, 4680-4691 (2013)

8. Zhang, L, Ahmad, B, Wang, G: The existence of an extremal solution to a nonlinear system with the right-handed Riemann-Liouville fractional derivative. Appl. Math. Lett. 31, 1-6 (2014)

9. Ahmad, B, Nieto, JJ: Riemann-Liouville fractional differential equations with fractional boundary conditions. Fixed Point Theory 13(2), 329-336 (2012)

10. Zhang, $X, L i u, L, W u, Y$ : The uniqueness of positive solution for a singular fractional differential system involving derivatives. Commun. Nonlinear Sci. Numer. Simul. 18, 1400-1409 (2013)

11. Zhang, X, Liu, L: Multiple positive solutions of a singular fractional differential equation with negatively perturbed term. Math. Comput. Model. 55, 1263-1274 (2012)

12. $\mathrm{Wu}, \mathrm{W}, \mathrm{Zhou}, \mathrm{X}$ : Eigenvalue of fractional differential equations with $p$-Laplacian operator. Discrete Dyn. Nat. Soc 2013, Article ID 137890 (2013) 
13. Zhang, $X, L i u, L, W u, Y$ : The eigenvalue problem for a singular higher fractional differential equation involving fractional derivatives. Appl. Math. Comput. 218, 8526-8536 (2012)

14. Wang, J, Xiang, H, Liu, Z: Positive solutions for three-point boundary value problems of nonlinear fractional differential equations with $p$-Laplacian. Far East J. Appl. Math. 37, 33-47 (2009)

15. Zhang, $X, L i u, L, W u, Y$ : Existence results for multiple positive solutions of nonlinear higher order perturbed fractional differential equations with derivatives. Appl. Math. Comput. 219, 1420-1433 (2012)

16. Zhang, $X, L i u, L$, Wiwatanapataphee, $B, W u, Y$ : Positive solutions of eigenvalue problems for a class of fractional differential equations with derivatives. Abstr. Appl. Anal. 2012, Article ID 512127 (2012)

17. Li, C, Luo, X, Zhou, Y: Existence of positive solutions of the boundary value problem for nonlinear fractional differential equations. Comput. Math. Appl. 59, 1363-1375 (2010)

18. Leibenson, L: General problem of the movement of a compressible fluid in a porous medium. Izv. Akad. Nauk Kirg. SSSR 9, 7-10 (1983) (in Russian)

19. Kilbas, AA, Srivastava, HM, Trujillo, J: Theory and Applications of Fractional Differential Equations. North-Holland Mathematics Studies, vol. 204. Elsevier, Amsterdam (2006)

20. Miller, K, Ross, B: An Introduction to the Fractional Calculus and Fractional Differential Equations. Wiley, New York (1993)

21. Podlubny, I: Fractional Differential Equations. Mathematics in Science and Engineering. Academic Press, New York (1999)

10.1186/1687-1847-2014-186

Cite this article as: Yang and Zhong: The uniqueness of solution for a fractional order nonlinear eigenvalue problem with $p$-Laplacian operator. Advances in Difference Equations 2014, 2014:186

\section{Submit your manuscript to a SpringerOpen ${ }^{\circ}$ journal and benefit from:}

- Convenient online submission

Rigorous peer review

- Immediate publication on acceptance

Open access: articles freely available online

- High visibility within the field

- Retaining the copyright to your article 